\title{
CASTIGO A LOS OFICIALISMOS Y CICLO POLÍTICO DE DERECHA EN AMÉRICA LATINA
}

Punishment of incumbents and

right-wing political cycle in Latin America

\author{
Juan Pablo Luna ${ }^{1}$ \\ Instituto de Ciencia Política \\ Universidad Católica de Chile \\ jlunaf@uc.cl \\ https://orcid.org/0000-0001-7597-203X \\ Cristóbal Rovira Kaltwasser ${ }^{2}$ \\ Escuela de Ciencia Política \\ Universidad Diego Portales \\ cristobal.rovira@mail.udp.cl \\ https://orcid.org/0000-0002-5453-3318
}

Recibido: 20/3/2021

Aceptado: 27/4/2021

Resumen: El artículo presenta una caracterización de tres estrategias (no electoral, electoral no partidaria, y partidaria) que la derecha emplea en América Latina actualmente. Analizando el derrotero reciente de los sistemas de partido en la región argumentamos también que el denominado giro a la derecha constituye un proceso de alternancia generado por el castigo a los oficialismos de la última década y media (mayoritariamente de izquierda), más que un realineamiento ideológico estructural. La alternancia se produce, además, en un contexto en que tienden a desaparecer o debilitarse significativamente los

1 Juan Pablo Luna agradece el apoyo del Fondo Nacional de Desarrollo Científico y Tecnológico de Chile (proyecto Fondecyt 1190345), del Instituto Milenio Fundamentos de los Datos, y del Instituto Milenio VioDemos.

2 Cristóbal Rovira Kaltwasser agradece el apoyo del Fondo Nacional de Desarrollo Científico y Tecnológico de Chile (proyecto Fondecyt 1180020), del Centro de Estudios de Conflicto y Cohesión Social (COES, ANID/ Fondap/15130009) y del Observatorio de Transformaciones Socioeconómicas (ANID / PCI / Max Planck Institute for the Study of Societies / MPG190012). 
partidos establecidos, y en el que irrumpen vehículos electorales usualmente evanescentes. Finalmente, argumentamos que no parece existir hoy espacio en la región - especialmente debido a la crisis social asociada a los efectos de la pandemia del COVID-19- para el fortalecimiento de una derecha neoliberal. Sin embargo, el contexto actual sí parece propicio para el surgimiento de outsiders de derecha, capaces de estructurar una agenda prorden que incorpore, en distintas proporciones, políticas de mano dura, conservadurismo valórico, y liberalismo de mercado.

Palabras clave: derecha, América Latina, partidos políticos, outsiders, alternancia.

Abstract: This article presents a characterization of three type of strategies (non-electoral, non-partisan electoral, and partisan) pursued by the right in contemporary Latin America. By analyzing the recent course of the party systems in the region, we also argue that the so-called turn to the right constitutes a process of power alternation generated by the punishment of incumbents of the last decade and a half (mostly on the left), rather than a structural ideological realignment. This alternation in power occurs in a context, in which established parties tend to disappear or become substantially weaker, and in which short-lived electoral vehicles are gaining traction. Finally, we argue that there does not seem to be space in the region today - especially because of the social crisis associated with the effects of the COVID-19 pandemic - for the strengthening of a neoliberal right. However, the current context does seem propitious for the emergence of right-wing outsiders, capable of structuring a pro-order agenda that incorporates, in different proportions, 'iron-hand' policies, value conservatism, and market liberalism.

Keywords: right, Latin America, political parties, outsiders, alternation in power. 


\section{Introducción}

A inicios del 2010 el debate político en América Latina estaba marcado por la discusión respecto al resurgimiento de la izquierda y la identificación de distintas variantes de centroizquierda en la región. En aquel entonces, nos propusimos observar qué estaba sucediendo del otro lado del espectro ideológico. Para ello organizamos un taller académico en la ciudad de Berlín que nos permitió, junto a varios colegas, desarrollar una perspectiva comparativa respecto a la situación de la derecha en distintos países de la región. A partir de dicha perspectiva finalmente editamos el libro La resiliencia de la derecha en América Latina (Luna y Rovira Kaltwasser, 2014). En este breve ensayo nos interesa retomar algunas de las ideas desarrolladas en ese libro y discutir su validez en el contexto actual.

Es importante señalar que el libro se articuló en torno a una conceptualización mínima de derecha. La conceptualización que allí ofrecemos, inspirándonos en la clásica distinción de Norberto Bobbio (2014), argumenta que una ideología política de derecha asume que las desigualdades en una sociedad son de carácter natural y, por tanto, que no corresponde a la intervención estatal contravenirlas. Con base en esta definición, argumentamos que a raíz de los altos niveles de desigualdad socioeconómica imperantes en América Latina las fuerzas de derecha deberían tener escasas posibilidades de lograr altos niveles de apoyo electoral en un contexto democrático. Aunque consistente con el clima imperante en la época, dicha expectativa teórica contravenía la experiencia histórica de la región. Al mismo tiempo, a pesar de su declive electoral, la derecha continuaba siendo muy relevante en las lógicas político-institucionales que observábamos en las sociedades latinoamericanas. En ese sentido, un argumento central de La resiliencia de la derecha América Latina es que aún en períodos de declive electoral y en contextos nacionales en que la derecha política es débil, los grupos conservadores recurren a diversos mecanismos para lograr influir en el proceso democrático y proteger así sus intereses y posturas ideológicas.

De tal manera, para comprender cómo es que la derecha se desenvuelve en un contexto que en principio le resulta desfavorable, propusimos analizar tres estrategias eventualmente disponibles para los sectores de derecha. En primer lugar, señalábamos la presencia de una derecha no electoral. Este tipo de manifestación tiene relación con la acción política sobre todo de sectores oligárquicos y (nuevos) grupos empresariales, vía la acción política directa. En este plano, la derecha no electoral cuenta con poder estructural y con poder instrumental como herramientas de acción política privilegiados (Fairfield, 2015; Hacker y Pierson, 2010; Winters, 2011; véase también Campello, 2015). Mientras el poder estructural permite a la derecha no electoral afectar el contexto (principalmente la eco- 
nomía) en que se hace política y se gobierna, el poder instrumental le permite a la derecha no electoral influir las decisiones de política pública y la competencia electoral vía acciones de lobby, financiamiento electoral y control de agenda en los medios.

En segundo lugar, identificábamos la posibilidad de la irrupción de una derecha electoral no partidaria, caracterizada por la irrupción de liderazgos con programa y acción de gobierno conservadora, no necesariamente anclados en un vehículo partidario consolidado. En este sentido, especulamos que un potencial resurgimiento de la derecha podría ir de la mano de la irrupción de fuerzas populistas que lograsen articular proyectos personalistas afines a los intereses de élites conservadoras, pero capaces también de lograr tracción electoral en segmentos significativos de la ciudadanía. En tercer y último lugar, identificábamos también la posibilidad de una rearticulación de la derecha electoral partidaria, en base a la permanencia y eventual crecimiento de partidos de derecha consolidados; o en función de un desenlace exitoso de los incipientes procesos de construcción partidaria por parte de intereses conservadores que se insinuaban en algunos países de la región.

En base a la identificación de estas tres estrategias, los distintos capítulos del libro mapeaban su prevalencia y contornos específicos en cada caso nacional. Al observar el panorama regional también argumentamos que el futuro de la derecha en la región dependería de su capacidad de hacer uso de manera individual o combinada de estas distintas estrategias, en oposición a los gobiernos de izquierda que predominaban en ese momento.

Sobre la base del marco conceptual introducido en La resiliencia de la derecha en América Latina, este ensayo propone interpretar el resurgimiento de fuerzas conservadoras en la región. Lo hacemos en función de cinco argumentos breves. Primero, especulamos acerca de la existencia de un giro a la derecha en la región y proponemos que más que un giro ideológico, estamos en presencia de un voto castigo en contra de oficialismos desgastados por su acción de gobierno. Segundo, analizamos brevemente las características de un rasgo permanente en la política de la región: la influencia política de los intereses conservadores en la sociedad a partir de estrategias de la derecha no electoral. Tercero, damos cuenta de los casos más relevantes respecto a la emergencia de expresiones de derecha electoral no partidaria. Cuarto, identificamos expresiones partidarias de la derecha en la región, analizando procesos de permanencia o construcción de nuevos partidos. Finalmente concluimos acerca de la naturaleza del giro a la derecha, y su atenuación, enfatizando las que parecen ser características emergentes de la competencia política contemporánea. Allí ofrecemos un panorama comparativo de dos patrones de competencia que han emergido alternativamente en distintos 
países: la desestructuración de los sistemas políticos nacionales, o la emergencia de un patrón polarizado de competencia entre dos fuerzas principales. Dichos patrones no son necesariamente estables, y en varios casos han emergido de modo secuencial. Especulamos finalmente sobre los posibles efectos sobre dichos patrones de competencia política de las consecuencias sociales de la pandemia del COVID-19.

\section{2. ¿Ciclo político de la derecha o castigo a los gobiernos de turno?}

La izquierda en América Latina se encuentra hoy debilitada, habiendo perdido terreno respecto a su consolidación a inicios del nuevo milenio. Por un lado, las así llamadas fuerzas de izquierda populista se encuentran en franca retirada (por ejemplo, Correa en Ecuador) o han desembocado en una deriva autoritaria (por ejemplo, Maduro en Venezuela y Ortega en Nicaragua). Por otro lado, los casos emblemáticos de la izquierda moderada o socialdemócrata han sufrido últimamente importantes derrotas electorales: ni el Partido de los Trabajadores en Brasil, ni el Partido Socialista en Chile, ni el Frente Amplio en Uruguay controlan ya el poder ejecutivo. Producto de esta difícil situación de la izquierda, han emergido en la región interpretaciones que plantean la presencia de un (contra) giro a la derecha. A modo de ejemplo, dicha interpretación cuenta con evidencia favorable en el éxito electoral reciente de las fuerzas de derecha que han logrado ganar elecciones en todos los países del Cono Sur (Macri en Argentina, Bolsonaro en Brasil, Piñera en Chile y Lacalle en Uruguay).

Las fuerzas de derecha han mantenido su preeminencia tradicional en Paraguay y Colombia, al tiempo que también han avanzado en algunos países centroamericanos como Honduras y Guatemala. En el caso de Colombia, la derecha tradicional ha dado paso, a partir de la emergencia y permanente centralidad del liderazgo de Uribe en el sistema, a una secuencia de liderazgos personalistas (Santos y Duque), aun cuando tal como señalan Rodríguez-Raga y Wills-Otero en su artículo en este número especial, el partido Centro Democrático está tratando de montar una organización partidaria que trascienda los personalismos. El caso de Ecuador, por su parte, registra un giro no electoral, en el sentido que el presidente Moreno, ungido como continuador del liderazgo de Correa, una vez en el poder se corre a la derecha. A su vez, el reciente triunfo del banquero Guillermo Lasso implica la aparición de una derecha electoral que, si bien es personalista, podría eventualmente dar paso a una organización partidaria (ver al respecto el artículo de Navia y Umpierrez de Reguero en este número especial). En el caso de Paraguay, la ANR (Partido Colorado), retoma el control del ejecutivo 
luego del interregno marcado por el trunco gobierno de Lugo, mientras que, en El Salvador, el liderazgo de derecha radical de Nayib Bukele desplazó, súbitamente, a uno de los sistemas bipartidistas más institucionalizados de la región (FMLN y ARENA). También es importante notar que fuerzas de izquierda lograron retomar el control del ejecutivo luego de interregnos de derecha, en Argentina, Bolivia (en que la derecha llega al poder irregularmente y es desplazada por vía electoral), y México.

La tabla 1 presenta un panorama, a nivel regional, del tipo y secuencia de alternancia registrada a nivel presidencial desde inicios de los 2000 hasta las elecciones más recientes en cada país. La tabla 2 intenta caracterizar los patrones de alternancia observados en términos ideológicos, considerando también el tipo de organización electoral que impulsa al liderazgo presidencial en cada caso. En este plano, identificamos tres tipos de organización: partidos establecidos (presentes en el país y con cierto grado de desarrollo orgánico y programático), fuerzas populistas, y vehículos electorales personalistas. Aunque esta última es una distinción eventualmente tenue, a diferencia de las fuerzas populistas, los vehículos personalistas se articulan en torno a un liderazgo individual, y logran avanzar en base a una coalición electoral eventualmente más efímera que las que logran articular las fuerzas populistas, ya que estas últimos por lo general logran generar apoyo tanto a nivel de la ciudadanía como en determinadas élites. El caso peruano, por ejemplo, registra desde el año 2000 una larga sucesión de liderazgos personalistas a nivel presidencial. Dichos liderazgos, eventualmente fraguan una coalición electoral ganadora (al decir de Zavaleta, 2014: una coalición de independientes), pero fracasan en asentar su poder. Al mismo tiempo, el sistema peruano ofrece alternancia sin alternativa (Vergara, 2018), en tanto, independientemente de las promesas electorales, la política pública registra importantes continuidades con el modelo neoliberal implementado bajo el régimen autoritario de Fujimori. 
Tabla 1. Evolución de los liderazgos presidenciales en América Latina

\begin{tabular}{|c|c|c|c|c|}
\hline País & $\begin{array}{c}\text { Izquierda } \\
\text { radical }\end{array}$ & $\begin{array}{l}\text { Izquierda } \\
\text { moderada }\end{array}$ & $\begin{array}{c}\text { Derecha } \\
\text { moderada }\end{array}$ & $\begin{array}{c}\text { Derecha } \\
\text { radical }\end{array}$ \\
\hline Argentina & & 2003-15, 2019- & 2015-19 & \\
\hline Bolivia & 2006-19, 2020- & & & \\
\hline Brasil & & $2003-16$ & 2018-18 & 2018- \\
\hline Chile & & $2000-10,2014-18$ & $\begin{array}{c}2010-14 \\
2018-\end{array}$ & \\
\hline Colombia & & & & 1991- \\
\hline Costa Rica & & 2014- & & \\
\hline Ecuador & $2007-17$ & & $2017-$ & \\
\hline El Salvador & & 2009-19 & & 2019- \\
\hline Guatemala & & $2008-12$ & 2012- & \\
\hline Honduras & 2006-09 & & & 2009- \\
\hline México & 2018- & & $2000-18$ & \\
\hline Nicaragua & 2007 & & & \\
\hline Panamá & & 2004-09, 2019- & 2014-19 & \\
\hline Paraguay & & $2008-12$ & $2012-$ & \\
\hline Perú & & & $2000-$ & \\
\hline $\begin{array}{l}\text { República } \\
\text { Dominicana }\end{array}$ & & 2000-04 & & \\
\hline Uruguay & & 2005-19 & 2020 & \\
\hline Venezuela & 1999- & & & \\
\hline
\end{tabular}

Fuente: Elaboración propia. 
Tabla 2. Trayectoria ideológica y organizacional reciente de los sistemas de partido latinoamericanos

Ideología política

\begin{tabular}{|c|c|c|c|c|c|}
\hline & & $\begin{array}{c}\text { Izquierda } \\
\text { radical }\end{array}$ & $\begin{array}{l}\text { Izquierda } \\
\text { moderada }\end{array}$ & $\begin{array}{c}\text { Derecha } \\
\text { moderada }\end{array}$ & $\begin{array}{c}\text { Derecha } \\
\text { radical }\end{array}$ \\
\hline & Partidos & & Brasil 2003-16 & Argentina & \\
\hline & políticos & & Chile & 2015-19 & \\
\hline & establecidos & & 2014-18 & Uruguay & \\
\hline & & & Uruguay 2005- & $2020-$ & \\
\hline & & & 19 & Chile 2010- & \\
\hline & & & El Salvador & $14,2018-$ & \\
\hline & & & 2009-19 & México & \\
\hline & & & Costa Rica & $2000-12$ & \\
\hline & & & 2014- & $\begin{array}{l}\text { Paraguay } \\
\text { 2012- }\end{array}$ & \\
\hline & $\begin{array}{l}\text { Fuerzas } \\
\text { populistas }\end{array}$ & $\begin{array}{l}\text { Venezuela } \\
\text { 2009- }\end{array}$ & $\begin{array}{l}\text { Argentina } 2003- \\
15,2019-\end{array}$ & & \\
\hline $\begin{array}{c}\text { Tipo de } \\
\text { organización } \\
\text { política }\end{array}$ & & $\begin{array}{l}\text { México 2018- } \\
\text { Nicaragua } \\
\text { 2007- }\end{array}$ & $\begin{array}{l}\text { República Do- } \\
\text { minicana 1996- }\end{array}$ & & \\
\hline & & $\begin{array}{l}\text { Ecuador 2007- } \\
17\end{array}$ & & & \\
\hline & & $\begin{array}{l}\text { Bolivia 2006- } \\
\text { 19, 2020- }\end{array}$ & & & \\
\hline & $\begin{array}{l}\text { Vehículos } \\
\text { electorales }\end{array}$ & $\begin{array}{l}\text { Honduras } \\
2006-09\end{array}$ & $\begin{array}{l}\text { Paraguay 2008- } \\
12\end{array}$ & $\begin{array}{l}\text { Ecuador } \\
\text { 2017- }\end{array}$ & $\begin{array}{l}\text { Brasil } \\
\text { 2018- }\end{array}$ \\
\hline & $\begin{array}{l}\text { (personalis- } \\
\text { tas) }\end{array}$ & & $\begin{array}{l}\text { Guatemala } \\
2008-12\end{array}$ & $\begin{array}{l}\text { México } 2 \\
012-18\end{array}$ & $\begin{array}{l}\text { Colombia } \\
\text { 1991- }\end{array}$ \\
\hline & & & $\begin{array}{l}\text { Panamá 2004- } \\
\text { 09, 2020- }\end{array}$ & $\begin{array}{l}\text { Guatemala } \\
\text { 2012- }\end{array}$ & $\begin{array}{l}\text { EI Salva- } \\
\text { dor 2019- }\end{array}$ \\
\hline & & & & Perú 2000- & Honduras \\
\hline & & & & $\begin{array}{l}\text { Panamá } \\
\text { 2014-19 }\end{array}$ & $2009-$ \\
\hline
\end{tabular}

Fuente: Elaboración propia. 
La tabla 2 señala en negrita los casos actualmente - a marzo de 2021 - en el gobierno de cada país. Si bien es posible observar un avance de las fuerzas de derecha respecto a décadas previas, se trata de un movimiento más bien atenuado. Dicho movimiento, además, tiene relación con el éxito de diferentes estrategias de movilización política (partidos establecidos, fuerzas populistas y vehículos electorales personalistas). En nuestra impresión, el giro a la derecha ha sido menos pronunciado que el pasado giro a la izquierda, y parece enfrentar problemas para consolidarse, al menos por el momento, como un ciclo político duradero de derecha. La relativa brevedad de la alternancia hacia la derecha en Argentina, la crisis del gobierno de Piñera en Chile, la creciente impopularidad de Bolsonaro en Brasil, y el rápido reemplazo por vía electoral del gobierno interino de Añez en Bolivia ilustran la endeblez electoral del giro a la derecha. En dicho sentido, parece razonable asumir que la alternancia refleja más dinámicas posicionales (oposición vs. oficialismo), que realineamientos masivos y significativos a nivel de los electorados nacionales.

Por eso mismo, las razones que explican la endeblez del giro posicional hacia la derecha afectan también a la izquierda, y responden a rasgos estructurales de la dinámica electoral que se ha ido consolidando en la región en los últimos años. Aunque profundizaremos este punto en la conclusión, es importante subrayar aquí que más que un nuevo ciclo político marcado por el ascenso de la derecha, la región ha venido experimentando procesos de alternancia electoral e ideológica pautada por el castigo a los oficialismos, en un contexto en que en general, los partidos políticos como organización, han ido perdiendo progresivamente capacidad de encapsular y movilizar consistentemente bases electorales relativamente estables (Luna y Munck, 2022; Luna et al., 2021). ¿Qué explica, desde esta perspectiva, el debilitamiento de la izquierda en la región y el relativo ascenso de liderazgos presidenciales de derecha?

Los mecanismos causales que explicarían el giro atenuado a la derecha son de dos tipos. Primero, la derecha logra avanzar electoralmente en el contexto de un desgaste de los oficialismos y, en particular, de aquellos asociados a la izquierda. El desgaste de los oficialismos se produce, a su vez, asociado a dos fenómenos específicos: el estancamiento económico y una creciente centralidad de sonados casos de corrupción asociados al neoextractivismo, la inversión en infraestructura durante el boom de los commodities, y el financiamiento irregular de la política (por ej., casos Odebretch y Lava Jato). En este sentido, el desgaste de las fuerzas gobernantes se produce en el marco de una economía política pautada por un creciente deterioro de las condiciones externas, y luego de una década larga de crecimiento y expansión económica asociada al boom de los commodities. El boom, así como el consenso de los commodities (Svampa, 2019), había asentado 
a los gobiernos previos, tanto por sus efectos directos sobre la economía y el crecimiento, como con base en el financiamiento de innovaciones de política social que habían logrado consolidar una reducción de la pobreza y la desigualdad en la región, y una expansión significativa del consumo (Lustig, 2017; CEPAL varios años).

Las crecientes restricciones económicas y la irrupción en la agenda pública de escándalos de corrupción deterioran así el apoyo de los sectores más volátiles del electorado a las fuerzas de izquierda. Este contexto también repercute en el interior de las fuerzas de izquierda, provocando tensiones y la eventual fragmentación entre grupos más moderados y otros más radicales. Dicha fragmentación y tensión interna abre oportunidades para el avance de coaliciones electorales de derecha y de liderazgos pragmáticos capaces de aumentar su caudal electoral mediante la moderación de su agenda ideológica. Así, por ejemplo, la evidencia empírica para el caso de Chile refleja que el éxito electoral de Piñera se vincula a una moderación programática de la derecha, en un escenario pautado por la fragmentación de la izquierda en diferentes partidos (Rovira Kaltwasser, 2019, 2020).

Segundo, la derecha logra avanzar electoralmente en un contexto pautado por la creciente centralidad de dos asuntos (issues) relativamente ortogonales al clivaje izquierda-derecha asociado a la dimensión económica. Por un lado, el auge económico de la región se asocia también a un aumento de la criminalidad y la violencia; dicho incremento se produce de modo relativamente homogéneo en la región, aunque parte de líneas de base heterogéneas (Bergman, 2018; Feldmann y Luna, 2022). La creciente centralidad de la criminalidad, potenciada también por su preponderancia en la pauta de noticias que desarrollan los medios de comunicación abre espacio para fuerzas de derecha que impugnan la incapacidad de los oficialismos para solucionar los temas de inseguridad. Este contexto termina siendo especialmente propicio para candidaturas que prometen mano dura como eventual solución a la crisis de inseguridad.

Por otro lado, ante los avances logrados por el progresismo con relación a la agenda de derechos de género y diversidad sexual, se consolida también una reacción conservadora en torno a temas morales. Dicha reacción se vincula, además, a la politización y creciente movilización electoral de sectores evangélicos (especialmente neopenteconstales), los que irrumpen con fuerza en distintos contextos electorales en la región (Smith y Boas, 2020). La movilización de los sectores evangélicos se consolida en casos en que históricamente contaba con fuerza electoral (por ej., en Brasil y Centroamérica), pero también, en casos en que la Iglesia católica (por ej. Chile) o un fuerte carácter secular (por ej., Argentina y Uruguay) resultaban centrales en términos de la articulación de la competencia política. 
Habiendo analizado las características del actual giro a la derecha en la región (tenue, sin cristalización en realineamientos duraderos, propiciado por el avance de liderazgos articulados en torno a estrategias electorales diversas) y los mecanismos que parecen haberlo propiciado (el desgaste de los oficialismos en un contexto pautado por el deterioro económico, escándalos de corrupción, la creciente preocupación por la criminalidad, y la movilización electoral de sectores conservadores en lo valórico), las próximas secciones dan cuenta tres tipos de articulación política de derecha: no electoral, electoral no partidaria, y electoral partidaria. En la conclusión volvemos a una reflexión general sobre esta ola de alternancia y su eventual persistencia.

\section{La derecha no electoral}

Tal como argumentamos más arriba, América Latina ha vivido en los últimos años un giro moderado hacia gobiernos de derecha. La derecha no electoral, asociada a los intereses económicos, ha mantenido su tradicional fortaleza, aún durante el ciclo de gobiernos de izquierda. La economía política del neoextractivismo y el denominado consenso de los commodities (Svampa, 2019) ilustran la capacidad constante de las élites económicas de incidir en procesos con consecuencias significativas respecto al modelo de desarrollo y sus implicancias distributivas. En dicho sentido, cabe observar, por ejemplo, la capacidad de los intereses empresariales de inhibir de modo sistemático, más allá de divergencias relevantes a nivel nacional (Falleti y Riofrancos, 2018), la protección de derechos de ciudadanía de comunidades vulnerables (pobres rurales e indígenas) aún bajo gobiernos con marcado discurso progresista (Luna y Munck, 2022). En este plano observamos, por tanto, más continuidad que cambio.

Al observar otras manifestaciones de derecha no electoral, sí es posible distinguir una serie de transformaciones relevantes. Por un lado, en cuanto al clivaje valórico, la Iglesia católica, bastión tradicional del conservadurismo moral en la región, ha perdido poder y capacidad de incidir en la política nacional. El declive de la Iglesia católica no es solo imputable al daño reputacional causado por la retahíla de escándalos en que se ha visto inmersa a nivel global, sino también a la creciente ineficacia de una estrategia de acción política centrada en mecanismos de influencia no electoral (mediante el lobby con élites políticas tradicionales, también declinantes en buena parte de la región). En este sentido, la Iglesia evangélica posee dos ventajas competitivas: un fuerte arraigo en sectores populares y una flexibilidad estratégica que le permite poner su capacidad de movilización electoral al servicio de liderazgos políticos conservadores emergentes. Así, la irrupción electoral de los sectores evangélicos ha generado réditos electorales a 
partidos establecidos de la región (el Partido Nacional en Uruguay, Renovación Nacional en Chile), a fuerzas populistas (Bolsonaro en Brasil, López-Obrador en México), y a liderazgos personalistas (Añez en Bolivia, J. A. Kast en Chile, y varios movimientos conservadores en Centroamérica).

Por otro lado, en algunos casos como el de Bolsonaro en Brasil, J. A. Kast en Chile, Bukele en El Salvador y Cabildo Abierto en Uruguay, el conservadurismo moral se ha vinculado también a una plataforma prorden que promete articular soluciones de mano dura para el problema de la seguridad pública. Este grupo de liderazgos, tácita o implícitamente, también recurre a un resorte tradicional de poder de la derecha latinoamericana, cuya centralidad política ha regresado a la agenda de la región: las FF. AA. y más ampliamente, las fuerzas de seguridad.

Los medios de comunicación han sido tradicionalmente otro de los mecanismos clave a través de los que la derecha no electoral ejerce poder de agenda en la región (Reis, 2011; Hughes y Prado, 2011). Aunque los medios mainstream de comunicación siguen siendo relevantes en este sentido, dos tendencias recientes parecen haber atenuado su capacidad de incidir directamente en la agenda política. En primer lugar, durante el ciclo progresista, una serie de países avanzaron con legislación tendiente a fortalecer el pluralismo de los medios de comunicación (Kitzberger, 2016). En segundo lugar, la disrupción tecnológica y la crisis de avisaje en los medios tradicionales ha potenciado también la irrupción de medios alternativos, es decir, ha potenciado eventualmente una diversificación de la oferta y una atomización en el consumo ciudadano de noticias en las sociedades latinoamericanas contemporáneas (Luna et al., 2021). Si bien los efectos de ambas transformaciones son incipientes, es de esperar que terminen limitando, al menos en los márgenes, la histórica capacidad de la derecha no electoral de controlar y limitar la agenda de políticas públicas. No obstante, también es cierto que las transformaciones de los medios de comunicación a nivel global, y particularmente la creciente centralidad de las redes sociales, generan un ambiente propicio para la circulación de noticias falsas, la microsegmentación de la movilización política y procesos de polarización social a partir de la consolidación de cámaras de eco (Keane, 2013). Estas herramientas pueden ser utilizadas por las fuerzas políticas, especialmente aquellas con mayor acceso a financiamiento (entre las que se encuentra la derecha), para desarrollar una movilización electoral eventualmente efectiva. 


\section{La derecha electoral no partidaria}

Lo propio de la derecha electoral no partidaria es que busca ganar votos mediante la defensa de ideas de derecha (ya sea en torno al libre mercado o posturas morales conservadoras), pero lo hace prácticamente sin énfasis alguno en la construcción de partidos políticos. En términos generales, dos son los mecanismos que la derecha electoral no partidaria suele utilizar: por un lado, la construcción de vehículos electorales personalistas que son controlados por un líder específico y, por otro lado, la formación de una coalición de apoyo para lograr conquistar el poder ejecutivo e intentar generar gobernabilidad. Aun cuando se trata de estrategias diferentes, en la práctica muchas veces terminan siendo combinadas, sobre todo cuando un líder personalista accede al poder y, por tanto, trata de implementar reformas para las que necesariamente requiere tener respaldo tanto de sectores de la élite como de la ciudadanía. A su vez, ambas estrategias usualmente van de la mano con el desarrollo de una retórica populista, que al establecer una distinción maniquea en el pueblo puro y la élite corrupta, logra conectar con el malestar de sectores del electorado que no se sienten representados por la clase política (Mudde y Rovira Kaltwasser, 2017).

Al observar el desarrollo político de Latinoamérica en los últimos años, es posible encontrar varios casos de derecha electoral no partidaria, que articulan posiciones tanto de derecha moderada como de derecha radical (ver tabla 2 más arriba). Sin embargo, nos parece relevante detenernos en dos casos que probablemente sean los dos ejemplos más emblemáticos de una nueva derecha radical en América Latina, que está muy en sintonía con la derecha radical a nivel global (al respecto, ver el artículo de Zanotti y Roberts en este número especial), a saber, José Antonio Kast en Chile y el Bolsonarismo en Brasil.

José Antonio Kast es un político de la derecha chilena que hizo su carrera al interior de la Unión Demócrata Independiente, pero que para la elección presidencial del año 2017 decidió postular como candidato independiente, presentándose como una suerte de outsider y levantando un programa de gobierno con una agenda de derecha populista radical (Rovira Kaltwasser, 2019). En cierto sentido, esto marca un punto de inflexión al interior de la derecha chilena, que desde la recuperación de la democracia a fines de los años 1980 ha venido apostando por la construcción de partidos políticos antes que por el levantamiento de vehículos electorales personalistas. Aun cuando José Antonio Kast moviliza a un sector reducido del electorado, su irrupción representa un serio problema para la derecha chilena, ya que esta última tiene crecientes dificultades para lograr mantener unidos aquellos grupos que presionan por moderación y los que buscan una mayor radicalización (Rovira Kaltwasser, 2020). Todo indica que esta tensión 
marcará los próximos años de la derecha chilena, puesto que José Antonio Kast ha inscrito un nuevo partido político (Acción Republicana, estudiado en el artículo de Campos en este número especial), con el que busca ganarse un espacio a nivel local y parlamentario mediante la promoción de una agenda centrada en tres temas centrales: defensa del modelo de libre mercado, adopción de valores morales tradicionales y propuestas de mano dura para combatir la delincuencia. En consecuencia, José Antonio Kast y su formación política representan un caso emblemático de un vehículo personalista de derecha populista radical que seguirá tensionando a los partidos de derecha convencional establecidos en el país y, por lo mismo, es crucial analizar las estrategias que estos últimos llevarán a cabo para lidiar con esta situación.

No cabe duda de que la irrupción de Jair Bolsonaro en Brasil representa el mayor éxito electoral de la derecha populista radical en América Latina. Si bien es cierto que Bolsonaro es un ejemplo de liderazgo personalista, a nuestro juicio es importante destacar que su acceso al poder y capacidad de mantenerse como presidente obedece en gran medida a la formación de una coalición de apoyo tanto a nivel de la ciudadanía como de las élites del país. Por un lado, la evidencia empírica respecto al triunfo electoral de Bolsonaro revela que su votación se explica por dos motivos principales: el desarrollo de una agenda moralmente conservadora y la politización de una identidad negativa hacia el Partido de los Trabajadores en particular y la clase política en general (Fuks, Ribeiro, Borba, 2020; Rennó, 2020). Por otro lado, el ascenso de Bolsonaro y su capacidad de mantenerse en el poder descansa también en la formación de una red de apoyo conservadora a nivel de élite, que se nutre por sobre todo de gran parte del empresariado, la Iglesia evangélica y las fuerzas armadas del país (Hunter y Power, 2019). Todavía es muy temprano para saber cómo evolucionará el bolsonarismo, pero este ejemplo revela que la irrupción de una derecha electoral no partidaria con una agenda de populista radical puede ganar terreno en América Latina, sobre todo si es capaz de concertar a sectores de la élite que estén dispuestos a respaldar un proyecto político de esta naturaleza.

\section{La derecha partidaria}

Las expresiones de derecha partidaria en América Latina contemporánea son relativamente escasas. Al día de hoy son tres los países actualmente gobernados por organizaciones partidarias consensualmente consideradas de derecha: Chile, Uruguay, y Paraguay. Se trata, además, de fuerzas caracterizadas como de centro-derecha o de derecha liberal. México fue gobernado por un partido tradicional de derecha (el PAN), durante dos sexenios, entre 2000 y 2012. Al término de 
ese sexenio, el PAN sufrió no solo las consecuencias del desgaste de gobernar el país durante la mayor escalada de violencia criminal registrada en su historia, sino también, de una erosión progresiva de sus enclaves electorales a nivel territorial. En Argentina, por otra parte, la derecha logró articularse (como no lo hizo durante todo el siglo xx) en torno a una de las pocas organizaciones partidarias que surgieron y lograron cierta permanencia en toda la región. Aunque poco duradero en términos de su control del gobierno nacional, la exitosa construcción del PRO, y su creciente capacidad de articularse como alternativa regular al peronismo, superando, además, la crisis de su liderazgo fundacional, ha logrado por el momento dotar de una estructura de competencia bipolar al sistema de partidos argentino (Vommaro, 2017, 2019).

Entre las derechas gobernantes, la ANR o el Partido Colorado de Paraguay, se caracteriza por una inusual capacidad de mantener la hegemonía electoral en el país. Dicha hegemonía, quebrada únicamente por la irrupción del liderazgo de Fernando Lugo, resulta de la capacidad de ofrecer una renovación permanente a nivel de liderazgos presidenciales, con una aceitada máquina electoral que logra traccionar y movilizar adhesiones sin contrapeso a nivel territorial. Dicha máquina se financia mediante el acceso a prebendas estatales, así como a través del acceso a financiamiento empresarial vinculado a sectores conservadores nacionales y a intereses extranjeros (particularmente brasileños).

Las derechas gobernantes en Chile y Uruguay poseen, por otro lado, similitudes relevantes. En ambos casos, los oficialismos de derecha corresponden a coaliciones electorales que integran varios partidos políticos (Renovación Nacional, la Udi, y EVopoli en el caso de Chile; el Partido Nacional (PN), el Partido Colorado (PC), el Partido Independiente (PI), el Partido de la Gente, y Cabildo Abierto en el caso de Uruguay). En el caso de Uruguay, cabe señalar que el Partido de la Gente es hoy un vehículo personalista en disolución, articulado en torno al liderazgo de un exitoso emprendedor. El Partido de la Gente no cuenta hoy con puestos en el gabinete y su único diputado es hoy independiente. Ambas coaliciones combinan también la presencia de partidos establecidos (RN y la UDI en Chile; el PN, el PC, y el PI en Uruguay) y nuevos partidos políticos (Evopoli en Chile; el Partido de la Gente y Cabildo Abierto en Uruguay). En ambos casos, los referentes tradicionales de la derecha presentan grados significativos de fragmentación interna. Si bien la fragmentación intrapartidaria es una clave de larga duración en la política uruguaya, el Partido Nacional asistió en las últimas elecciones al surgimiento de dos nuevas tendencias que tensionaron su interna (el liderazgo de Alonso, vinculado también al mundo evangélico; y el liderazgo de Sartori, un outsider con perfil empresarial, que logró desplazar a referentes históricos del partido). El Partido Colorado, por su parte, vivió un fuerte proceso de renovación mediante 
el surgimiento de una tendencia también nueva, nucleada en torno al liderazgo de Talvi, quien logró desplazar en la interna al liderazgo histórico de Sanguinetti. No obstante, a poco andar, Talvi, el líder de Ciudadanos, y entonces Canciller de la República, decidió abandonar la política dejando el control de un muy debilitado Partido Colorado, nuevamente en manos de Sanguinetti. En Chile, Renovación Nacional se encuentra quebrado internamente por pujas fraccionales y entre sus liderazgos más visibles. Mientras tanto, EVOPOLI surge como partido en torno a liderazgos jóvenes vinculados al primer gobierno de Piñera, pero también en torno a debates sobre la renovación de la centro-derecha, parcialmente disparados por procesos de crisis interna de la UDI. La crisis de la UDI también se vincula a escándalos referidos al financiamiento electoral y la vinculación del partido con el lobby empresarial.

Ambas coaliciones de derecha llegaron al gobierno explotando el desgaste de sus antecesores y promoviendo una agenda de derecha liberal que enfatizaba propuestas de reactivación económica y seguridad pública. En el caso de Chile, la coalición se estructuró en torno al liderazgo de Piñera, quien ya había ejercido la presidencia entre 2010 y 2014. No obstante, Piñera compitió en ambos casos con base en su posicionamiento como un empresario hábil, capaz de promover el crecimiento económico y la eficiencia en la gestión. En Uruguay, Lacalle es un político profesional, cuya carrera se desarrolla completamente en el ámbito político y partidario.

Otra diferencia relevante entre ambos casos es que, la coalición chilena excluye, en términos de su composición, a un nuevo partido de extrema derecha consolidado en torno al liderazgo de José Antonio Kast: el Partido Republicano. Como indicamos más arriba, ese partido promueve una agenda conservadora en lo valórico (asociada a sectores evangélicos), una propuesta neoliberal en lo económico (con entonaciones abiertamente libertarias), y una propuesta de mano dura vinculada a una reivindicación frecuente de las FF. AA., Carabineros, y su actuar durante la dictadura encabezada por Augusto Pinochet. Cabildo Abierto en Uruguay, un partido liderado por figuras directamente vinculadas a las FF. AA., posee características similares a las del Partido Republicano chileno. No obstante, Cabildo Abierto tiene participación directa en la coalición de gobierno, tanto a través de su bancada parlamentaria como en función del control de una serie de ministerios y puestos clave en el ejecutivo. En ambos casos, sin embargo, los nuevos partidos de derecha radical desafían actualmente la hegemonía electoral de los partidos de centro-derecha que actualmente lideran los gobiernos de Chile y Uruguay. 


\section{Conclusiones}

En nuestra impresión, el ciclo de alternancia hacia la derecha observado en América Latina se asocia, en términos de su dinámica causal, a un movimiento electoral pautado por el descontento con los oficialismos (mayoritariamente de izquierda) que gobernaron la región desde mediados de los 2000. Gran parte de este descontento se explica por deterioro del crecimiento económico una vez que se agotó el commodity boom, así como al desgaste causado por escándalos de corrupción. Por su parte, la aparición de una agenda pública que le otorga creciente relevancia a problemas de seguridad y al conservadurismo valórico ofrecen posibilidades de crecimiento electoral a liderazgos y partidos de derecha.

Ahora bien, este ciclo de alternancia a la derecha se produce en un contexto general en que continua y se profundiza un proceso pautado por el declive de los partidos establecidos y la irrupción de nuevos vehículos electorales, la gran mayoría de los cuales resultan efímeros, más allá de su éxito electoral a corto plazo. Tal vez la única excepción a esta regla la constituya, en el ámbito de las expresiones de derecha, el caso del pro en Argentina (Vommaro, 2017, 2019). En un contexto de volatilidad partidaria, es dable esperar que los alineamientos electorales sean también volátiles, y que se torne el ciclo de alternancia a la derecha eventualmente más endeble. Dicha endeblez debería ser aún mayor en el marco del profundo proceso de crisis económica disparado por las consecuencias de la pandemia del Covid-19 y que en teoría deja poco espacio para la promoción de una agenda neoliberal, sino que más bien facilita el apoyo de una mayor intervención económica por parte del Estado. Visto así, dado que todo indica que la pandemia trae consigo un aumento de la desigualdad y la pobreza, se refuerza la idea central de La resiliencia en América Latina, en donde planteábamos que la derecha tiene pocas posibilidades de ganar elecciones en la región a través de recetas libremercadistas.

No obstante, las duras consecuencias socioeconómicas de la pandemia también podrían crear las condiciones propicias para la consolidación de una coalición prorden, que termine favoreciendo expresiones de derecha radical o, incluso, retrocesos autoritarios. En efecto, un aumento de la pobreza y la desigualdad puede desencadenar una creciente demanda por seguridad ciudadana en vastos sectores de la ciudadanía, sobre todo si la centroizquierda no es capaz de desarrollar políticas públicas frente a esta materia y si pone excesivo énfasis en temas de corte posmaterial que no necesariamente van a ser primordiales para aquellos grupos sociales que más fuertemente se ven afectados por las consecuencias socioeconómicas de la pandemia. 
Un escenario eventualmente alternativo al de la dilución de los sistemas de partido, lo constituye la creciente polarización entre fuerzas de izquierda y derecha. En aquellos países en que observamos niveles altos y crecientes de polarización, como Argentina y eventualmente Uruguay, pero también Bolivia (entre el MAS y los sectores conservadores) y Brasil (en caso que el PT logre rearticularse como principal oposición al liderazgo de Bolsonaro) es posible observar una estructura de competencia electoral ideológicamente más cristalizada. Este escenario resulta posible siempre y cuando las fuerzas de centro-derecha y centro-izquierda logren encontrar caminos de cooperación, ya que si uno de los dos bloques - $\mathrm{O}$ ambos - mantiene(n) una fragmentación interna, resulta difícil que la polarización se cristalice y potencialmente dé paso tanto a un mayor alineamiento electoral como a potenciales ciclos de recambio político marcado por castigo a los gobiernos de turno. Sin embargo, estructuras similares a las observadas en este conjunto de casos han sido rápidamente desplazadas por la irrupción de outsiders. Los casos de López-Obrador en México y de Bukele en El Salvador ilustran este último escenario. 


\section{Referencias}

Bergman, M. (2018). More money, more crime: Prosperity and rising crime in Latin America. Oxford, Reino Unido: Oxford University Press.

BobBio, N. (2014). Derecha e izquierda. Madrid, España: Taurus.

CAmpello, D. (2015). The politics of market discipline in Latin America: globalization and democracy. Cambridge, Reino Unido: Cambridge University Press.

Cepal. (Varios años). Panorama Social de América Latina. Santiago, Chile: Cepal.

FAirfield, T. (2015). Private wealth and public revenue. Cambridge, Reino Unido: Cambridge University Press.

Falleti, T. G., y T. N. Riofrancos (2018). Endogenous participation: Strengthening prior consultation in extractive economies. World Politics, 70(1), 86-121.

Feldmann, A., y J. P. LunA (2022). Criminal Governance and the Crisis of Contemporary Latin American States. Annual Review of Sociology, 48.

Fuks, M., E. Ribeiro y J. Borba (2021). From Antipetismo to Generalized Antipartisanship: The Impact of Rejection of Political Parties on the 2018 / Vote for Bolsonaro. Brazilian Political Science Review, 15(1), 1-28.

Hacker, J. S., y P. Pierson (2010). Winner-take-all politics: How Washington made the rich richer - and turned its back on the middle class. New York, EE. UU.: Simon and Schuster.

Hughes, S. y P. Prado (2011). Media diversity and social inequality in Latin America. En M. Blofield (ed.): The great gap: Inequality and the politics of redistribution in Latin America (pp. 109-146). Pensilvania, EE. UU.: University Park: Penn State University Press.

Hunter, W. y T. Power (2019). Bolsonaro and Brazil's Illiberal Backlash. Journal of Democracy, 30(1), 68-82.

Keane, J. (2013). Democracy and Media Decadence. Cambridge, Reino Unido: Cambridge University Press.

Kitzberger, P. (2016). Media wars and the new left: Governability and media democratisation in Argentina and Brazil. Journal of Latin American Studies, 48(3), 447-476.

Luna, J. P., y C. Rovira Kaltwasser (eds.). (2014). The Resilience of the Latin American Right. Baltimore, EE. UU.: The Johns Hopkins University Press. 
Luna, J. P., y C. Rovira Kaltwasser (2021). A la derecha de la derecha: ¿hay espacio para una fuerza populista radical de derecha? Fundación Friedrich Ebert: Montevideo.

LunA, J. P., y G. Munck (2022). Introduction to Contemporary Latin American Politics: The Quest for Democracy and Citizenship Rights. Cambridge, Reino Unido: Cambridge University Press.

Luna, J. P., R. Piñeiro, F. Rosenblatt y G. Vommaro (2021a). Diminished Parties. Democratic Representation in Contemporary Latin America. Cambridge, Reino Unido: Cambridge University Press.

Luna, J. P., S. Toro y S. Valenzuela. (2021b). Amplifying Counter-public Spheres on Social Media: News Sharing of Alternative vs Traditional Media After the 2019 Chilean Uprising (documento inédito).

Lustig, N. (2017). El impacto del sistema tributario y el gasto social en la distribución del ingreso y la pobreza en América Latina: Argentina, Bolivia, Brasil, Chile, Colombia, Costa Rica, Ecuador, El Salvador, Guatemala, Honduras, México, Nicaragua, Perú, República Dominicana, Uruguay y Venezuela. Una aplicación del marco metodológico del proyecto Compromiso con la Equidad (CEQ). El trimestre económico, 84(335), 493-568.

Mudde, C., y C. Rovira Kaltwasser (2017). Populism. A Very Short Introduction. Oxford, Reino Unido: Oxford University Press.

Rennó, L. (2020). The Bolsonaro Voter: Issue Positions and Vote Choice in the 2018 Brazilian Presidential Elections. Latin American Politics \& Society, 62(4), $1-23$.

Rovira Kaltwasser, C. (2020). El error de diagnóstico de la derecha chilena y su encrucijada actual. Revista de Estudios Públicos, 158, 31-59

Rovira Kaltwasser, C. (2019). La (sobre)adaptación programática de la derecha chilena y la irrupción de la derecha populista radical. Colombia Internacional, 99, 29-61.

REIS, E. (2011). Elite perceptions of poverty and inequality in Brazil. En M. Blofield (ed.). The great gap: inequality and the politics of redistribution in Latin America (pp. 89-109). Pensilvania, EE. UU.: University Park: Penn State University Press.

Smith, A. E., y T. C. BoAs. (2020). Religion, Sexuality Politics, and the Transformation of Latin American Electorates. APSA Annual Meeting and exhibition. Democracy, difference, and destabilization. Artículo presentado en el congreso anual de la American Political Science Association. Seattle, EE. UU. 
Svampa, M. (2019). Neo-extractivism in Latin America: socio-environmental conflicts, the territorial turn, and new political narratives. Cambridge, Reino Unido: Cambridge University Press.

Vergara, A. (2018). Ciudadanos sin república. Lima; Perú: Planeta.

Vommaro, G. (2017). La larga marcha de Cambiemos: la construcción silenciosa de un proyecto de poder. Buenos Aires: Siglo xxi Editores.

Vommaro, G. (2019) De la construcción partidaria al gobierno: PRo-Cambiemos y los límites del «giro a la derecha» en Argentina. Colombia Internacional, 99, 91-120.

Winters, J. A. (2011). Oligarchy. Cambridge, Reino Unido: Cambridge University Press.

Zavaleta, M. (2014). Coaliciones de independientes: Las reglas no escritas de la política electoral. Lima, Perú: Instituto de Estudios Peruanos.

Los autores han realizado igual contribución al artículo y son los únicos responsables de su contenido. 
